\title{
Plant Landscape Planning Based on the Concept of Biodiversity Conservation
}

\author{
Dr. Shivali Kharoliwal \\ Lzebra College Kota, Rajasthan, India \\ kharoliwalshivali76@gmail.com
}

\begin{abstract}
A survey was conducted in University campus to review the floristic diversity of Angiosperms along with their medicinal use. The survey included observation and field visit of Kota University campus. a whole of 103 medicinal plants were identified. Most species belonged to Fabaceae followed by Moraceae. The foremost purpose of this survey is to assemble data about Medicinal plants available in campus. This study is concentrated to produce an effective knowledge of medicinal properties of plants so on to cure disease with nonside-effects.
\end{abstract}

Keywords: Ethnobotany, floristic, indigenous system, medicinal plants

\section{Introduction}

Vast ethnobotanical knowledge exists in India from ancient time. Since the 1950s the study of ethnobotany has intensified Ethnobotany deals with study and evaluation of plant resources After food and shelter man has developed remedies for curing various diseases. Traditional medicines have been used for thousands of years for treating various diseases. Although flora of Rajasthan has been compiled by various workers, there is increasing demand for herbal medicines. World Health Organisation (WHO) has emphasised the fact that more than $90 \%$ of world's population are exclusively dependent upon indigenous and traditional plant knowledge (1, 2). Deforestation, urbanization, industrialization, transmigration, colonization and other developmental activities have threatened not only biological resource but also ethnobotanical knowledge (3). Rajasthan is rich in plant resources. Kota, a city located in southeast of northern state of Rajasthan It is located about 240 kilometres (149 mi) south of the state capital, Jaipur, situated on the banks of Chambal River is also rich in biodiversity. University of Kota is located near Kabir circle. The present study highlights ethnomedicinal plants of Kota university campus.

\section{Methodology}

In order to determine floristic diversity in the study area we used reconnaissance method and survey was done on foot in different seasons of the year. The study site was divided into five blocks in such a way that all portions of university campus is covered eastern, western, northern, southern and central part of the study site. At each location quadrats of appropriate size were laid to assess the distribution pattern and plant species were recorded $(4,5,8)$. The families were classified according to Benthem and Hooker Classification (1862-1883). Identifications were done with the help of different floras and herbaria of Rajasthan. $(6,7)$.

\section{Results and discussion}

In the present study of Kota university campus 73 plant species are reported. Out of 73 plant studied, 63 species belong to dicotyledons and 10 to monocotyledons (Table1). Enumerated plants were dispersed among Leguminoseae (16species), Poaceae (10 species), Asteraceae and lamiaceae (5 species), Apocynaceae (4 species), Amaranthaceae, Acanthaceae, Asclepidiaceae, Meliaceae, Brassicaceae, Solanaceae ( 2 species) and remaining families (one species in each) were gathered from university campus. All the studied plant species have been arranged alphabetically along with their binomial, family, local name and their medicinal use. After careful screening many species have been recognized as important medicinal plants with various properties.

There is urgent need to conserve traditional medicinal plants as they are widely used in various cultural and religious rites and rituals, this would also help to create database regarding the flora of Kota district

Table 1: List of Angiosperm plant species along with their family, localname, habit and medicinal uses

\begin{tabular}{|c|c|c|c|c|c|}
\hline S.no. & Botanical name & Family & Local name & Habit & Medicinal uses \\
\hline 1. & Abrusprecatorius L. & Fabaceae & Chirmathi, rati & Climber & Cough. cold, Hair growth, demulcent \\
\hline 2. & $\begin{array}{c}\text { Acacia auriculiformis (A.Cunn). } \\
\text { Benth. }\end{array}$ & Mimosaceae & Ear leaf acacia & Large tree & Analgesic \\
\hline 3. & Acacia leucophloea (Roxb.) Wild. & Mimosaceae & Ronj & Large tree & $\begin{array}{c}\text { Antipyretic, antidote for snake bite, } \\
\text { expectorant }\end{array}$ \\
\hline 4. & Acacia nilotica (L.) Wild. Ex Del. & Mimosaceae & Desi kikar & Large tree & $\begin{array}{c}\text { Antimicrobial.antiplasmodial, HIV } \\
\text { treatment }\end{array}$ \\
\hline 5. & Acacia senegal (L.) Wild. & Mimosaceae & $\begin{array}{c}\text { Khairi, gum } \\
\text { arabic }\end{array}$ & $\begin{array}{c}\text { Medium size } \\
\text { tree }\end{array}$ & $\begin{array}{c}\text { Bronchitis, cough, diarrhea, } \\
\text { typhoidfever, upper respiratory tract } \\
\text { infections }\end{array}$ \\
\hline 6. & Acacia tortilis(Forssk.) Hayne. & Mimosaceae & Totluskikar, & Large tree & Skin allergy, cough, inflammatory \\
\hline
\end{tabular}




\begin{tabular}{|c|c|c|c|c|c|}
\hline & & & israilebabool & & reactions \\
\hline 7. & Achyranthes aspera L. & Amaranthaceae & $\begin{array}{l}\text { Putkanda, } \\
\text { Ultakanta }\end{array}$ & Annual herb & $\begin{array}{l}\text { Haemorrhids, indigestion, cough, } \\
\text { asthma, anaemia, jaundice, snakebite }\end{array}$ \\
\hline 8 & AdhatodavasicaL. & Acanthaceae & Basaka, Aroosa & Annual herb & Leukoderma, vomiting, heart troubles \\
\hline 9. & Aegle marmelos(L.) Corr. Serr. & Rutaceae & Belpathar & Large tree & Dysentery and diabetes \\
\hline 10. & Ageratum conyzoides $\mathrm{L}$. & Asteraceae & JangliPudina & Annual herb & $\begin{array}{l}\text { Burns, eye problems, pneumonia, uterine } \\
\text { disorders }\end{array}$ \\
\hline 11. & Ailanthus excelsaRoxb. & Simarubiaceae & Olooneem & Large tree & Asthma, bronchitis and dysentry \\
\hline 12. & Albizialebbeck(L.)Benth. & Mimosaceae & Siris & Large tree & Eye flue, gingivitis \\
\hline 13. & ApludamuticaL. & Poaceae & - & Herb & Disinfectant, healing minor wounds \\
\hline 14. & Alstoniascholaris(L.) R.Br. & Apocyanaceae & $\begin{array}{l}\text { Sapt-Parna, } \\
\text { Devil tree }\end{array}$ & Large tree & Lung infection, cough, asthma \\
\hline 15. & Amaranths viridis $\mathrm{L}$. & Amaranthaceae & Chaulai & Annual herb & $\begin{array}{l}\text { Diabetes, dysentery, urinary and eye } \\
\text { disorders }\end{array}$ \\
\hline 16. & ArgemonemaxicanaL. & Papaveraceae & $\begin{array}{c}\text { Jhaljai, } \\
\text { satyanashi }\end{array}$ & $\begin{array}{l}\text { Ephemeral } \\
\text { herb }\end{array}$ & Constipation, malaria \\
\hline 17. & Aristidaadscensionis $\mathrm{L}$ & Poaceae & & Herb & $\begin{array}{l}\text { Lactation stimulant, prevent itch and } \\
\text { ringworm }\end{array}$ \\
\hline 18. & AzadirachtaindicaA.Juss. & Meliaceae & Neem & Large tree & $\begin{array}{c}\text { Treats fungal infections, mosquito } \\
\text { repellant }\end{array}$ \\
\hline 19. & BarleriaprinotisL. & Acanthaceae & Pila Basa & Annual herb & Whooping cough, urinary infections \\
\hline 20. & Bauhinia variegata $\mathrm{L}$. & Caesalpiniaceae & Kachnar & $\begin{array}{c}\text { Medium size } \\
\text { tree }\end{array}$ & $\begin{array}{l}\text { Anti helminthic, antileprotic, antidote for } \\
\text { snake bite }\end{array}$ \\
\hline 21. & Blumealacera(Burm. f.) DC Prodr. & Asteraceae & JangliMooli & Annual herb & Bronchitis, blood diseases \\
\hline 22. & BoerhaaviadiffusaL. & Nyctaginaceae & $\begin{array}{c}\text { Santi, } \\
\text { Punarnava }\end{array}$ & $\begin{array}{c}\text { Annual } \\
\text { prostrate herb }\end{array}$ & Kidney disorder, insominia \\
\hline 23. & BrachiariadistachyaL. & Poaceae & & Herb & Soil binder \\
\hline 24. & Brachiaria ramose $\mathrm{L}$. & Poaceae & & Herb & Remidation of contaminated soil \\
\hline 25. & Butea monosperma(Lam.) Taub. & Fabaceae & Dhak & Large tree & Leprosy, gout, eye disease \\
\hline 26. & Calotropis gigantea (L.) W.T.Aiton. & Asclepiadaceae & Aak & $\begin{array}{l}\text { Perennial } \\
\text { shrub }\end{array}$ & Elephantiasis, nausea, vomiting \\
\hline 27. & Calotropisprocera(Ait.) Aiton. & Asclepiadaceae & Aak & $\begin{array}{l}\text { Perennial } \\
\text { shrub }\end{array}$ & Constipation, stomach ulcers \\
\hline 28. & Capparis decidua (Forssk.) Edgew. & Capparaceae & Kair, Tint & Small tree & Asthma, toothache, cough \\
\hline 29. & Cassia fistula $\mathrm{L}$. & Caesalpiniaceae & Amaltas & Large tree & Antiseptic, laxative \\
\hline 30. & Cassia obtusifoliaL. & Caesalpiniaceae & Pavad & Herb & Anti -helminthic, stomach ache \\
\hline 31. & Cassia siamea(Lam.) Irwin et Barneby & Caesalpiniaceae & $\begin{array}{l}\text { Seemia, } \\
\text { Kasood } \\
\end{array}$ & $\begin{array}{c}\text { Medium size } \\
\text { tree }\end{array}$ & Anti- cancer, antidepressant \\
\hline 32. & Catharanthus roseus (L.) G.Don. & Apocyanaceae & Sadabahar & Herb & Stomach ache, relieves muscle pain \\
\hline 33. & CenchrusciliarisL. & Poaceae & Anjan & Herb & Body pain and menstural disorder \\
\hline 34. & Cocculushirsutus(L.) Diels. & Manispermaceae & Faridbuti & Twiner herb & Eczema, dyspepsia \\
\hline 35. & CorchorustrilocularisL. & Tiliaceae & Wild jute & Annual herb & Reduce swelling, nausea \\
\hline 36 & Coronopusdidymus(L.) Sm & Brassicaceae & Janglihaloon & Annual herb & Asthma, bronchitis \\
\hline 37 & Cynodondactylon(L.) Pers. & Poaceae & Doobghas, & Runner herb & Laxative, coolant, expectorant \\
\hline 38 & DaturainnoxiaMill. & Solanaceae & Datura & Annual herb & $\begin{array}{l}\text { Improves fertility, enhance heart } \\
\text { functions }\end{array}$ \\
\hline 39 & Delonixregia(Boj. ex. Hook.) Raf. & Caesalpiniaceae & Gulmohar & Large tree & Constipation, inflammation \\
\hline 40 & Eleusine indica $\mathrm{L}$. & Poaceae & Crowfoot grass & Herb & Antipyretic agent \\
\hline 41 & Euphorbia hirtaL. & Euphorbiaceae & Dudhi & Annual herb & Dysentery, jaundice, digestive problems \\
\hline 42 & Evolvulusalsinoides(L.) L. & Convolvulaceae & Vishnukrantha & Annual herb & Loss of memory, epilepsy, leukoderma \\
\hline 43 & FicusbenghalensisL. & Moraceae & Bar, Bargad & Large tree & Ulcers, vaginal complaints, leprosy \\
\hline 44 & FicusinfectoriaL. & Moraceae & Pilkhan & Large tree & Anti bacterial, anti diabetic \\
\hline 45 & FicusrelegiosaL. & Moraceae & peepal & Large tree & Skin complexion, nausea, vomiting \\
\hline 46 & Furcraeafoetida(L.) Haw. & Asparagaceae & $\begin{array}{l}\text { Mauritius } \\
\text { Hemp }\end{array}$ & $\begin{array}{c}\text { Perennial } \\
\text { cultivated } \\
\text { herb }\end{array}$ & Anti oxidant compounds \\
\hline 47 & GlinuslotoidesL. & Molluginaceae & Gandhi-buti & Annual herb & Anti helminthic \\
\hline 48 & Hibiscus rosa sinensis $\mathrm{L}$. & Malvaceae & Gurhal & Small tree & Hair loss, inflammation \\
\hline 49 & IndigoferalinnaeiAli. & Fabaceae & neel & Annual herb & Epilepsy, wound sores \\
\hline 50 & Jatropha curcasL. & Euphorbiaceae & Danti & Small tree & cancer \\
\hline 51 & Lantana camara L. & Varbenaceae & Lantana & Annual shrub & High blood pressure, eczema \\
\hline 52. & Leucascephalotes(Roth.) Spreng. & Lamiaceae & $\begin{array}{c}\text { Dadghal, } \\
\text { Draun pushpin } \\
\end{array}$ & Annual herb & Jaundice, cough, cold \\
\hline
\end{tabular}




\begin{tabular}{|c|c|c|c|c|c|}
\hline 53 & LindenbergiaruderalisKuntze. & Scrophulariaceae & Linderbergia & Annual herb & Chronic bronchitis, sore throat \\
\hline 54 & MeliaazadirachL. & Meliaceae & Banyan & Large tree & Rodenticidal, insecticidal \\
\hline 55 & Melothriamaderaspatana(L.)M.Roem & Cucurbitaceae & Aankhphod & Climber herb & Antidiabetic, larvicidal \\
\hline 56 & Murrayakoenigii(L.) Spreng. & Rutaceae & Kadhipatta & Small tree & Snakebite, piles \\
\hline 57 & OcimumamericanumL. & Lamiaceae & Nagadbavchi & Annual herb & Rheumatism, renal colic \\
\hline 58. & Ocimum sanctum L. & Lamiaceae & Tulsi & Herb & Bronchitis, asthma, malaria \\
\hline 59 & OcimumbasilicumL. & Lamiaceae & $\begin{array}{c}\text { Tulsi, sweet } \\
\text { basil }\end{array}$ & Herb & Bronchitis, asthma, malaria, cough \\
\hline 60 & OcimumtenuifoliumL. & Lamiaceae & Tulsi, holi basil & Herb & Bacterial infections, malaria, cough \\
\hline 61. & PortulacapilosaL. & Portulacaceae & Lunkhi & Annual herb & Diuresis, analgesia \\
\hline 62. & Saccharum officinarum L. & Poaceae & Ganna & Herb & Haemorrhage, anuria \\
\hline 63. & SaccharumravennaeL. & Poaceae & Sarkanda & Herb & biofuel \\
\hline 64. & Setariaviridis(L.) & poaceae & greenfoxtail & Herb & Diuretic, emollient \\
\hline 65. & Sidacordifolia L. & Malvaceae & Kharinti & Annual herb & Muscle cramp, skindisorder \\
\hline 66. & Sisymbriumirio L. & Brassicaceae & Khoobkalan & Annual herb & Cough and congestion \\
\hline 67. & Solanumsurattense Burm. f. & Solanaceae & Katehli & Annual herb & Fever, diarohhea \\
\hline 68. & Sonchus asper (L.) Gars. & Asteraceae & Dudhi & Annual herb & Menstural and eye problems \\
\hline 69. & Tephrosiaperpurea(L.) Pers. & Fabaceae & Jhojhru & Annual herb & Antihelminthic, antipyretic \\
\hline 70. & ThevetianeriifoliaJuss. & Apocyanaceae & Pila Kaner & Small tree & Emetic, loosen bowels \\
\hline 71. & Tridaxprocumbens L. & Asteraceae & Sadahari & Annual herb & Anti fungal, insect repellant \\
\hline 72. & Xanthium strumariumL. & Asteraceae & Maskhara & Annual herb & laxative \\
\hline 73. & Zizyphusjujuba Mill. & Rhamnaceae & Ber & $\begin{array}{c}\text { Medium size } \\
\text { tree }\end{array}$ & Stimulates apetite \\
\hline
\end{tabular}

\section{Conclusions}

Conservation of Medicinal plant is vital to avoid any further loss. Most of the medicinal plants were harvested from wild. Shrubs were dominant growth forms followed by herbs and trees. Depletion of indigenous knowledge among the people of the study area was serious due to disinterest of young generation to gain the knowledge. Finally to conclude this research article will attract the attention of ethno botanist, physiochemist and pharmacologist for further critical investigation of medicinal plants present in the campus.

\section{Acknowledgement}

I wish to thank Dr. Surabhi Shrivastav, Co-ordinator and Head, Department of Wildlife Science for her blessings.

\section{References}

[1] WHO, Traditional Medicine: Growing Needs and Potentials, 2002.

[2] WHO, "Traditional medicine, " 2012, http://www.who.int/mediacentre/factsheets/fs134/en.Vi ew at: Google Scholar

[3] WHO, "Traditional medicine, " 2008, http://www.who.int/mediacentre/factsheets/fs34/en.Vie w at: Google Scholar

[4] A. Sofowora, Medicinal Plants and Traditional Medicine in Africa, John Wiley \& Sons, New York, NY, USA, 1982.

[5] H. L. Park, H. S. Lee, B. C. Shin et al., "Traditional medicine in China, Korea, and Japan: a brief introduction and comparison, " Evidence-Based Complementary and Alternative Medicine, vol. 2012, Article ID 429103, 9 pages, 2012.View at: Publisher Site | Google Scholar

[6] L. Sharma (2006). Ethnobotany of Dang region in Rajasthan.
[7] S.Sharma and B.Tiagi (1979) Flora of North-East Rajasthan, Kalyani Publishers, New-Delhi.

[8] N.Mishra and A.Pareek (2015) Floristic Diversity of Angiosperms with special reference to their medicinal properties from Kota district of Rajasthan, India. 